\title{
Statistical and Image Analysis for Characterizing Simulated Atomic-Scale Damage in Crystals
}

\author{
D. $\mathrm{Li}^{1}$, B.J. Reich ${ }^{2}$ and D.W. Brenner ${ }^{1^{*}}$ \\ ${ }^{1}$ Department of Materials Science and Engineering \\ North Carolina State University, Raleigh, NC 27695-7907, USA \\ ${ }^{2}$ Department of Statistics \\ North Carolina State University, Raleigh, NC 27695, USA
}

*brenner@ncsu.edu

\begin{abstract}
While molecular dynamics simulations have been used for decades to study structure and formation mechanisms of plastic damage in crystals, the analytical tools needed to characterize collections of plastic defects have been limited. Here we demonstrate the use of two methods, spatial cross-correlations (CC) and Linear Discriminate Analysis (LDA), to analyze and compare plastic damage profiles among molecular dynamics simulations in which damage was created by straining bi-crystals containing symmetric tilt grain boundaries with different tilt angles. Two potentials were used, one representing $\mathrm{Cu}$ and one representing $\mathrm{Ag}$, and two coarse-grained descriptors for different types of crystal damage were used, averaged central symmetry parameters (CSP) and atomic hydrostatic stress (HS). We find that in general the CSP is a more accurate descriptor than HS for both analysis methods, and for data base sizes of about 30 or more simulations per tilt angle, the LDA does considerably better in predicting angle and material than the CC method. For example, at the largest data base size of 50 simulations per tilt angle and using the average CSP values, the LDA predicts the exact initial tilt angle and material type for $92 \%$ of the simulations, while the CC approach drops to $58 \%$. If the average HS is used instead of the average CSP, the LDA and CC predictions drop to $63 \%$ and $32 \%$, respectively. These results point to a number of possible applications of this method, for example in quantifying how the range of damage for a set of strained systems may depend on strain rate or temperature, or quantifying similarities between complex damage from processes such as indentation and energetic ion bombardment.
\end{abstract}

Key words: molecular dynamics; statistical analysis; plastic damage; dislocations 


\section{Introduction}

Molecular dynamics (MD) simulations have produced insights into plastic damage in crystals that have complemented, confirmed and in some cases revealed new structures and processes with respect to traditional materials defect theories.[1] MD simulations have also been used to probe the effects of conditions like temperature, applied stress and high strain rates on plastic deformation.[2-4] One of the major challenges to effectively using MD is interpreting the simulation data (e.g. atomic positions, velocities and forces) in a manner that produces useful insights. For molecular and cluster structures, a number of methods have been developed for analyzing complex shapes.[5-12] Some of these methods use direct statistical analysis to discriminate between structures, while others use statistical comparisons to structural databases. For crystals, a number of schemes have been introduced that can efficiently identify defects such as shear bands, stacking faults and dislocations from atom coordinates.[13-21] However, at present there are limited analysis tools that are comparable to those used for molecular and cluster systems that can efficiently identify and sort collections of defects (i.e. atomic analogues of a microstructure).[17,18]

In two recent studies, statistical and image analysis approaches were used to characterize plastic damage from MD simulations.[22,23] Both studies used coarse-grained matrices of central symmetry parameters (CSPs) [13] generated from a training set of plastic damage in strained bi-crystals containing two complementary symmetric grain boundaries with different tilt angles prepared as described in Section II below. The first study used three spatial regression approaches, a conditional autoregressive model, discrete wavelets, and principle component analysis, in an attempt to predict plastic damage at different tilt angles based on the training set.[22] Although the statistical models varied in their predictive capabilities, none was able to predict spatially-resolved plastic damage at an acceptable level to replace an MD simulation. In the second study, normalized spatial autocorrelation matrices were created from the CSP matrix of each MD simulation, and then cross-correlation (CC) matrices were generated from each of the auto-correlation matrices.[23] Using the largest element of the CC matrices as

a predictor, the method was able to correctly connect the plastic damage in each simulation to within $+/-5^{\circ}$ of the initial tilt angle for $87 \%$ of the simulations based on a data base of $30 \mathrm{MD}$ simulations at each tilt angle.

Motivated by the need for better tools for analyzing complex atomic defect structures, and encouraged by our previous results,[23] we report in the present paper results from a more comprehensive set of analyses using the set of atomic systems of strained bicrystals. This new study expands on the prior work in several important ways. First, in addition to the image analysis approach used previously, we apply Linear Discriminate Analysis (LDA) to the MD simulation data.[24] This method is applied to the coarse grained representations of the data through a rank transformation followed by Discrete Fast Fourier Transform (DFFT), and does not require calculating CC matrices. Second, in addition to the $\mathrm{Cu}$ potential used previously, the simulations use a second interatomic potential fit to the properties of $\mathrm{Ag}$. This allows us to 
determine whether the analyses methods can discriminate between the types of material, and is a first step toward quantifying differences in collections of atomic-level defects in terms of material property. Third, the calculations are repeated using hydrostatic stress (HS) instead of CSP to generate the coarse grained matrices, followed by the same statistical and image analyses. These calculations were done to explore how sensitive the results are to the damage descriptors taken from the simulations. Finally, the MD simulations are repeated for a larger number of runs. This allows us to characterize how the predictive capabilities of the analyses methods depend on data base size.

\section{Computational Methods}

The MD simulations were carried out using the LAMMPS code[25] and interatomic forces taken from Embedded-Atom Method potentials for $\mathrm{Cu}$ and Ag.[26,27] The initial bicrystals were generated from two face-centered cubic (fcc) lattices with lattice constants for $\mathrm{Cu}$ and $\mathrm{Ag}$ rotated around the [100] direction and truncated to create two complementary tilt grain boundaries in the $x$-y plane. For tilt angles of $0^{\circ}$ and $90^{\circ}$ (both of which are ideal crystals without a grain boundary), the $x-y$ interface is along a (001) and (011) plane, respectively. Atoms were removed along the grain boundaries that were less than about $80 \%$ of the nearneighbor distance, and the dimensions of the periodic simulation box were chosen as multiples of the rotated supercell dimensions. Each system contained between 377,260 and 484,549 atoms, and had dimensions ranging from $16.67 \mathrm{~nm}$ to $18.89 \mathrm{~nm}$ for $\mathrm{Cu}$ and between $18.45 \mathrm{~nm}$ and $21.41 \mathrm{~nm}$ for $\mathrm{Ag}$.

After the atomic configurations were generated, the systems were relaxed to minimize the potential energy. This was followed by equilibration to zero pressure and a temperature of $300 \mathrm{~K}$ using the Nose'-Hoover method for 100 ps.[28-30] To generate plastic damage, the systems were strained by scaling atom positions and the box length in the $z$ direction by a constant engineering strain up to a total strain of 0.1 over 100,000 time steps (100 ps). During this process the Nose'-Hoover method maintained a temperature of 300K and an average zero pressure in the $x$ and $y$ directions. To generate multiple plastic damage configurations for each tilt angle, velocities from the initially equilibrated system for each tilt angle and the two elements were replaced with new velocities taken from a Boltzmann distribution at $300 \mathrm{~K}$. The systems were then re-equilibrated for 2 ps at $300 \mathrm{~K}$ and zero pressure, followed by the same straining procedure. This was repeated 50 times for each tilt angle and both elements for a total of $50 \times 19 \times 2=1900$ straining simulations.

The centro-symmetry parameter is defined as

$$
C S P=\sum_{i=1}^{\frac{N}{2}}\left|r_{i}-r_{i+N / 2}\right|^{2}
$$

where $r_{i}$ and $r_{(i+N / 2)}$ are vectors from the central atom to a pair of opposite neighbors. [13] For perfect fcc crystals, the two vectors cancel because of symmetry and the CSP values are zero. Thermal vibrations and the presence of defects increases CSP values. For example, in $\mathrm{Cu}$ 

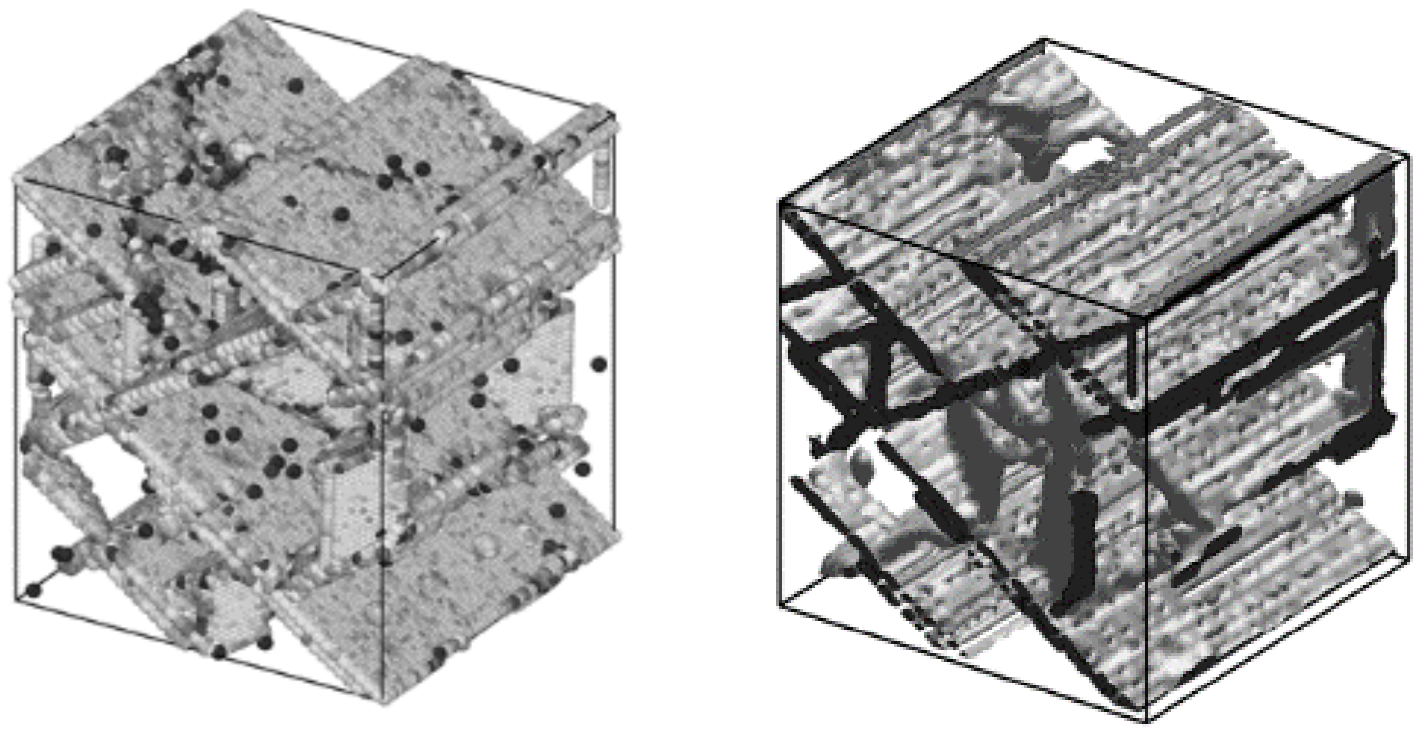

Figure 1: Representations of the final atomic configuration from a MD simulation after straining starting with a tilt of $90^{\circ}$. (A) Atomistic scale, where only atoms with a CSP greater than 2 are shown. (B) The same configuration after coarse graining. Only voxels with average CSP values greater than 2 are shown.

simulations at $300 \mathrm{~K}$ for non-defected regions the atoms have values of $\mathrm{CSP}<2, \mathrm{CSP}=4-5$ for atoms adjacent to vacancies, CSP $=5$ for atoms in dislocation loops, and CSP $=5-6$ for atoms in stacking faults. The HSs were calculated in the standard manner used in MD simulations. [25]

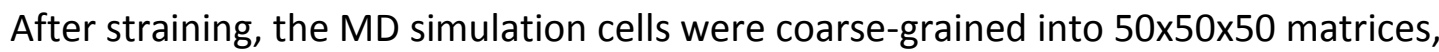
and each matrix element was assigned the average CSP values or the average HS values for each atom contained in that region of the simulation box. To ensure that matches are not a result of the grain boundary structure instead of the plastic damage, the element values in the eight rows of the CSP matrix containing atoms from the grain boundaries (fours rows per boundary) were set to zero. With this degree of coarse graining each box contained on average 3-4 atoms. This procedure facilitates the analysis, helps to average the CSP and HS values, and reduces the total amount of data to be analyzed. Plotted in the left panel of Figure 1 is the final atomic configuration from an example MD simulation that started with a tilt angle of $90^{\circ}$ prior to straining. Only atoms with a CSP greater than 2 are shown. The right panel illustrates the coarse grained matrix generated from the atomic configuration. It is clear from the images that the coarse-grained matrix is a reasonable representation of the simulation result.

\section{Spatial Correlation Analysis}

The spatial CC approach used here is taken from image analysis and supervised pattern recognition,[31] and should not be confused with time correlation functions that are more typically used to extract vibrational, transport and other time-dependent quantities from MD 
simulations.[32] The correlation matrix $C(n, m, l)$ of two matrices $f(i, j, k)$ and $g(i, j, k)$ is a moving dot product of the form

$$
C(n, m, l)=\sum_{i=1}^{N} \sum_{j=1}^{N} \sum_{k=1}^{N} f(i, j, k) * g(I, J, K)
$$

where

$$
I=\bmod (i+n, N), J=\bmod (j+m, N), K=\bmod (k+l, N) .
$$

The modification to the sums via Eq.(3), which is referred to as a circular CC, takes into account the periodic boundaries of the MD simulations. An autocorrelation matrix is generated when $f(i, j, k)=g(i, j, k)$, while a CC matrix is generated when $f(i, j, k) \neq g(i, j, k)$. To ensure that large values of the $C$ matrix indicate an offset where there is a relatively strong pattern matching, the elements in the $f$ and $g$ matrices are normalized by subtracting the mean and dividing by the standard deviation.

Normalized auto-correlation matrices were generated for each MD simulation from their corresponding CSP and HS matrices. CC matrices were then generated for each pair of normalized auto-correlation matrices for the CSP and HS data (CCs between the CSP and HS data were not generated). We note that without normalization a coarse-grained matrix with larger mean values would more likely have larger CC values regardless of the degree of pattern matching. There are a total of 19 angles, 50 runs per angle and two elements ( $\mathrm{Ag}$ and $\mathrm{Cu}$ ), which leads to the generation of a total of $19 * 50 * 2$ autocorrelation matrices for the CSP and for the HS values. There are therefore a total of [(19*50*2) $\left.)^{2}-2 * 19 * 50 * 2\right]$ CC matrices for the CSP and the same number of matrices for the HS. Taking advantage of the periodicity of the systems and the convolution theorem, three-dimensional discrete fast Fourier transforms (DFFT) were used to calculate all of the correlation matrices, which significantly shortened the required computational time compared to directly using Eqs. (2) and (3).

The largest value within the CC matrices for either the CSP or HS values was used to determine a match for a given configuration within the remainder of the data base, and hence predictions of the starting tilt angle for $2 \times 19 \times 50=1900$ trajectories. For example, if the largest value of each $\mathrm{CC}$ matrix for a given MD simulation is to another MD simulation that started with a tilt angle of $40^{\circ}$, the prediction is that the MD simulation being analyzed started with that angle. The accuracy of this approach can therefore be quantified by comparing the predictions to the actual starting grain boundary angles.

\section{Linear Discriminant Analysis}

LDA[24] is applied to the CSP and HE matrices after a rank transformation followed by a DFFT, and does not involve generating any correlation matrices. Let $Y_{i j}$ be the observation (either CSP or HS) in matrix element $j$ from simulation $i$, and $Y_{i}=\left(Y_{i 1}, \ldots, Y_{i n}\right)$ be the entire array of observations for simulation $i$. We first transform the data using a rank transformation. That is, if $Y_{i j}$ is greater than $100 p_{i j} \%$ of the observations in the entire dataset (all $i$ and $j$ ) then 
we set $Y_{i j}^{*}=\Phi^{-1}\left(p_{i j}\right)$ where $\Phi$ is the standard normal distribution function so that the transformed data $Y_{i}^{*}=\left(Y_{i 1}^{*}, \ldots, Y_{i n}^{*}\right)$ is approximately standard normal. We then take the DFFT of each array $Y_{i}^{*}$ to decorrelate the spatial observations and to capture periodic features. The DFFT output after extracting the unique real and imaginary parts is denoted as $Z_{i}=$ $\left(Z_{i 1}, \ldots, Z_{\text {in }}\right)$.

To classify simulation $i$, we use all simulations other than simulation $i$ to estimate the mean and standard deviation for each combination of DFFT term, angle, and material type. Let $\bar{Z}_{\text {lam }}$ be the sample mean for DFFT term $l \in\{1,2, \ldots, n\}$, angle $a \in\{0,5, \ldots, 90\}$, and material type $m \in\{C u, A g\}$, and $s_{\text {lam }}$ be the corresponding sample standard deviation. Given these estimates, the probability that simulation $i$ is angle $a$ and material type $m$ is

$$
Q_{i a m}=\frac{q_{\text {iam }}}{\sum_{u=0}^{90} \sum_{v=C u}^{A g} q_{i u v}}
$$

where $q_{\text {iam }}=\prod_{l=1}^{n} \varphi\left(Z_{i l} ; \bar{Z}_{\text {lam }}, s_{\text {lam }}\right)$ and $\varphi(\cdot ; \mu, \sigma)$ is the normal density function with mean $\mu$ and standard deviation $\sigma$. The probability that simulation $i$ corresponds to angle $a$ is the sum of material types, $\sum_{m} Q_{i a m}$, and the probability that simulation $i$ is of material type $m$ is the sum over angles, $\sum_{a} Q_{\text {iam }}$.

\section{Results and Discussion}

Given in Tables I and II are the probabilities for connecting each MD simulation to the original tilt angle and material type determined as described above using the coarse-grained CSP and HS data, respectively. In each table the data is broken down by the two analysis methods, as well as the accuracy by which each method can predict the exact angle and the angle within $+/-5^{\circ}$ independent of material type, the accuracy by which each method can predict the material type independent of angle, and the accuracies with which the two methods can predict both material type and angle. This data is further broken down by the number of runs in the data base (the column denoted "10 runs" used the first set of $10 \mathrm{MD}$ runs, the column denoted "20 runs" used the first 10 MD runs plus the second 10, etc.). This same data is also plotted in Figure 2.

There are several interesting trends apparent in this data in Table I and Figure 2(A). First, for all of the data the LDA shows a relatively large dependence on the number of runs up to about 30 runs per angle, after which the LDA probabilities start to level off. In contrast, the CC approach shows a much weaker data base size dependence. For all of the data except for the angle within $+/-5^{\circ}$ independent of material, the CC approach produces results that have better (or equivalent) accuracies than the LDA for the smallest data base size. By $30 \mathrm{MD}$ simulations per angle, however, LDA is more accurate than the CC approach for all data categories. Above this number of runs, the LDA is able to predict all data categories to better than $90 \%$, with a $99 \%$ prediction accuracy for angle within $+/-5 \%$ independent of material for the largest data 
base size. From the data it is clear that these methods are able to discriminate both initial tilt angle and material type.

Table I: Probabilities for matching angle and material type with the two methods using the CSP data.

\begin{tabular}{|c|l|c|c|c|c|c|}
\hline Method & \multicolumn{1}{|c|}{ Data Type } & 10 runs & 20 runs & 30 runs & 40 runs & 50 runs \\
\hline \multirow{4}{*}{$\begin{array}{c}\text { Cross } \\
\text { Correlation }\end{array}$} & Exact angle & 0.64 & 0.69 & 0.71 & 0.74 & 0.75 \\
\cline { 2 - 6 } & Angle within $+/-5^{\circ}$ & 0.88 & 0.93 & 0.94 & 0.94 & 0.95 \\
\cline { 2 - 7 } & Correct material & 0.67 & 0.72 & 0.72 & 0.73 & 0.74 \\
\cline { 2 - 7 } & Correct material \& exact angle & 0.47 & 0.52 & 0.54 & 0.56 & 0.58 \\
\cline { 2 - 7 } & Correct material \& angle within +/- $5^{\circ}$ & 0.60 & 0.66 & 0.68 & 0.69 & 0.73 \\
\hline \multirow{4}{*}{$\begin{array}{c}\text { Linear } \\
\text { Discriminant } \\
\text { Analysis }\end{array}$} & Exact angle & 0.43 & 0.85 & 0.95 & 0.96 & 0.97 \\
\cline { 2 - 7 } & Angle within $+/-5^{\circ}$ & 0.94 & 0.99 & 0.99 & 0.99 & 0.99 \\
\cline { 2 - 7 } & Correct material & 0.61 & 0.89 & 0.93 & 0.94 & 0.94 \\
\cline { 2 - 7 } & Correct material \& exact angle & 0.15 & 0.74 & 0.90 & 0.91 & 0.92 \\
\cline { 2 - 7 } & Correct material \& angle within $+/-5^{\circ}$ & 0.59 & 0.88 & 0.93 & 0.93 & 0.94 \\
\hline
\end{tabular}

Table II: Probabilities for matching angle and material type with the two methods using the HS data.

\begin{tabular}{|c|l|c|c|c|c|c|}
\hline Method & Data Type & 10 runs & 20 runs & 30 runs & 40 runs & 50 runs \\
\hline \multirow{4}{*}{$\begin{array}{c}\text { Cross } \\
\text { Correlation }\end{array}$} & Exact angle & 0.36 & 0.37 & 0.39 & 0.39 & 0.40 \\
\cline { 2 - 7 } & Angle within $+/-5^{\circ}$ & 0.58 & 0.59 & 0.59 & 0.61 & 0.62 \\
\cline { 2 - 7 } & Correct material & 0.67 & 0.67 & 0.68 & 0.68 & 0.68 \\
\cline { 2 - 7 } & Correct material \& exact angle & 0.29 & 0.29 & 0.32 & 0.30 & 0.32 \\
\cline { 2 - 7 } & Correct material \& angle within $+/-5^{\circ}$ & 0.42 & 0.42 & 0.44 & 0.44 & 0.45 \\
\hline \multirow{4}{*}{$\begin{array}{c}\text { Linear } \\
\text { Discriminant } \\
\text { Analysis }\end{array}$} & Exact angle & 0.12 & 0.30 & 0.42 & 0.57 & 0.66 \\
\cline { 2 - 7 } & Angle within $+/-5^{\circ}$ & 0.41 & 0.60 & 0.72 & 0.80 & 0.86 \\
\cline { 2 - 7 } & Correct material & 0.51 & 0.63 & 0.74 & 0.83 & 0.91 \\
\cline { 2 - 7 } & Correct material \& exact angle & 0 & 0.13 & 0.30 & 0.49 & 0.63 \\
\cline { 2 - 7 } & Correct material \& angle within $+/-5^{\circ}$ & 0.16 & 0.34 & 0.51 & 0.67 & 0.79 \\
\hline
\end{tabular}

Based on the data in Table II and the corresponding plot Figure 2(B), generating the same probabilities from the HS data shows some different trends and levels of accuracy. For example, while the results from the $\mathrm{CC}$ are better than those generated from the LDA at the smallest number of runs per angle, the accuracies from the CC analysis show relatively little improvement with data base size. In contrast, the LDA accuracies appear to increase roughly linearly and do not appear to reach a maximum with data base size. Both are different from the LDA analysis on the CSP matrices. For almost all of the predictions at each data base size, the CSP matrices yield more accurate values for both the CC and LDA methods than those from the $\mathrm{HS}$ matrices. The lone exception is the prediction of the correct material at the smallest data base size, where the CC method yields the same accuracy for both the CSP and HS matrices. 

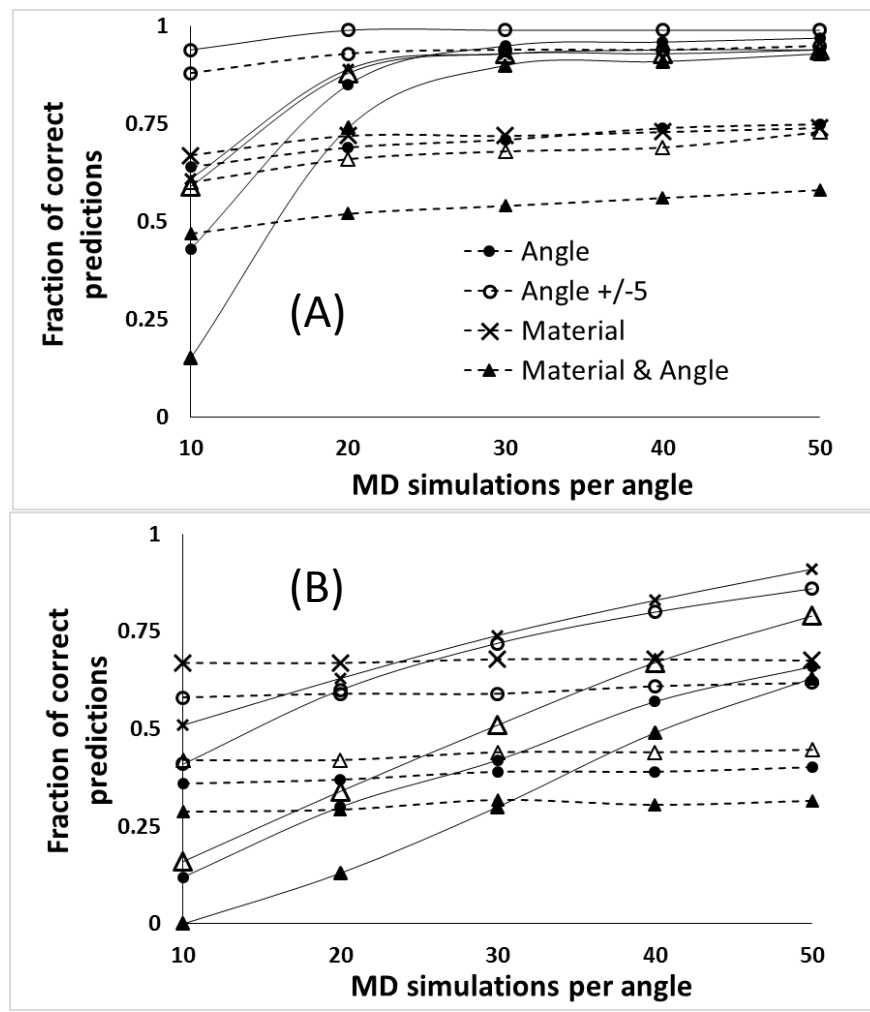

Figure 2: Plotted data from Tables I and II. For both plots the solid and dashed lines correspond to the LDA and CC approaches, respectively. (A) Data from the CSP matrices given in Table I. (B) Data from the HS matrices given in Table II. The correspondence between symbol and prediction for both plots is indicated in (A).
Plotted in Figures 3 and 4 are distributions of the difference between the predicted matching tilt angle and the actual tilt angle for each MD simulation, accumulated over all angles for different data base sizes using the CSP matrices. For simplicity only the Cu simulations are considered, and the distributions are normalized by the total number of matches for each data base size. Figure 3 is for the CC method and Figure 4 is for the LDA approach. As expected from the data in Tables I and II, for both methods the width of the distributions decreases with increasing data base size. However, at a data base size of $10 \mathrm{MD}$ runs per angle, the largest difference between the LDA predictions and the actual angle is $+/-10^{\circ}$, while the CC approach has seven errors that are larger than $+/-25^{\circ}(3.7 \%$ of the total). As the data base size increases, some of the improvement in predictive capability of the CC approach comes from the elimination of these large errors. For example, the percentage of errors in the initial tilt angle of magnitude $25^{\circ}$ or more for $\mathrm{MD}$

simulation data base sizes of $20,30,40$ and 50 drop to $0.53 \%, 0.35 \%, 0.26 \%$ and $0.21 \%$, respectively. For the LDA, the improvement in predictive capability comes from a decrease in the width of the distribution around the correct angle up to a data base size of $30 \mathrm{MD}$ runs per angle, after which the predictions level off to high values with an expected slight decrease in distribution width. Hence not only are the predictive capabilities different, the probability of large errors are also very different between the CC approach and LDA. Unlike CC, LDA requires estimating many parameters, namely the mean and variance for each combination of material, angle, and DFFT term. When the number of samples is small, these estimates are imprecise, which may explain LDA's poor predictive performance. As the number of samples increases, these estimates stabilize and performance improves.

To further explore some of the nuances of the CC approach, four example MD simulations that were generated with the Cu potential were chosen, one from each set of the simulations for initial tilt grain boundary angles of $10^{\circ}, 30^{\circ}, 50^{\circ}$ and $80^{\circ}$. The same procedure 

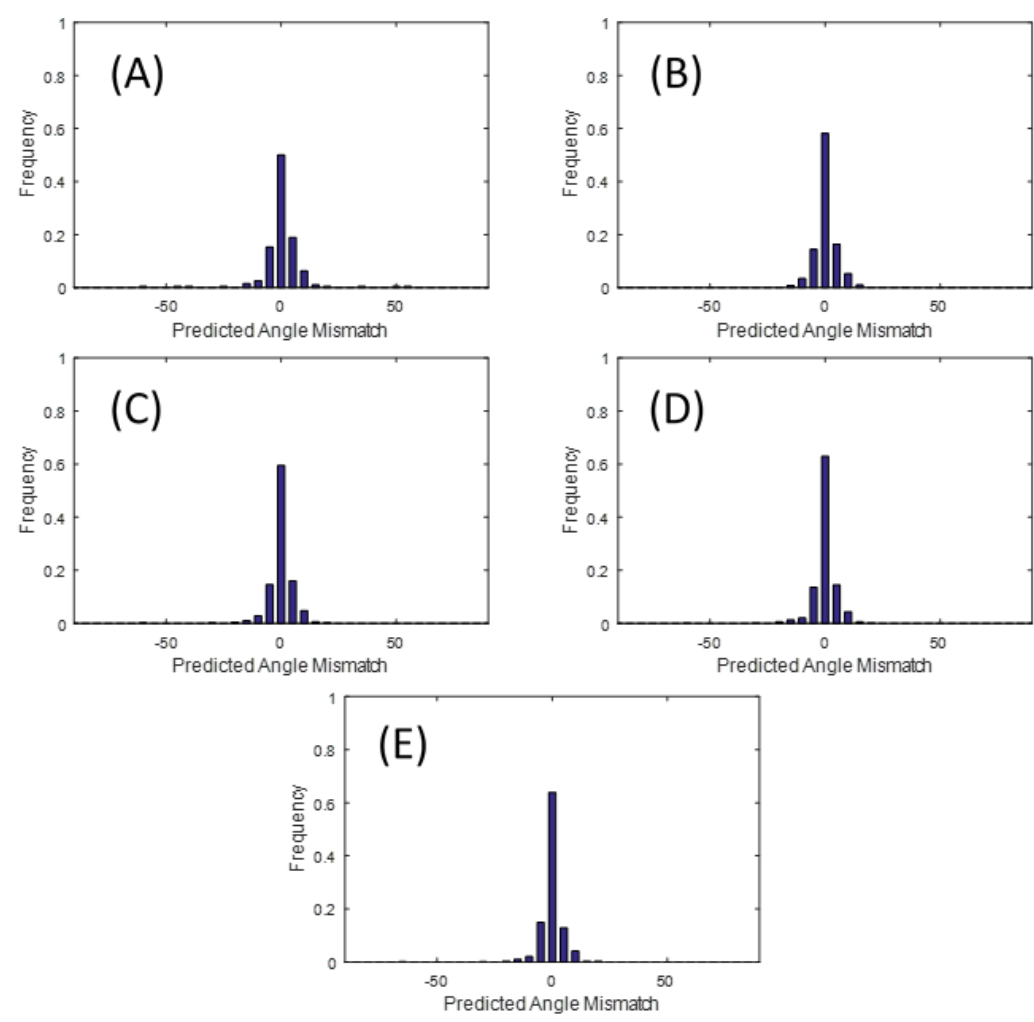

Figure 3: Distribution of the difference between the predicted initial grain boundary tilt angle and the actual angle calculated using the CC approach. (A)-(E): Data base sizes of 10, 20, 30, 40 and $50 \mathrm{MD}$ simulations per angle, respectively. was followed for MD simulations generated with the Ag potential, but the conclusions were the same as the Cu simulations so they are not discussed. Plotted in Figure 5 are the largest correlation coefficients from the CC matrices between each example simulation and the other simulations at the same angle. These were generated using the data base of 20 runs per angle for each element. The closed symbols correspond to a CC coefficient for a Cu MD simulation while the open symbols correspond to $\mathrm{Ag}$ MD simulations. From the plot it is clear that there are a range of correlation coefficients within each angle, and that these ranges

significantly overlap both in terms of MD simulations with the $\mathrm{Cu}$ versus $\mathrm{Ag}$ potential at the same angle, and for angles that are different from the matching angle. Despite this overlap, for the example angles of $10^{\circ}, 50^{\circ}$ and $80^{\circ}$, the largest CC coefficient matches the angle of the run and the material, i.e. the predicted initial tilt angle is correct. For the $30^{\circ}$ example run, however, the largest CC coefficient occurs for a simulation with an initial tilt angle of $20^{\circ}$ and using the $\mathrm{Ag}$ potential. Hence for this randomly-chosen example MD simulation the method misses both the angle and material. As discussed above, as the data base of MD simulations increases, the probability for matching angle and material increases. This is not because the range of correlation coefficients decreases for each angle with an increasing data base size, but rather the probability of a simulation at the correct angle and material better matching a given simulation increases with data base size.

\section{Conclusions}

While MD simulations have been used for decades to study structure and formation mechanisms of plastic damage in crystals, the analytical tools needed to characterize collections of plastic defects have been limited.[17,18] This is in contrast to MD simulations of soft materials and clusters, where a number of structure analysis tools have been 

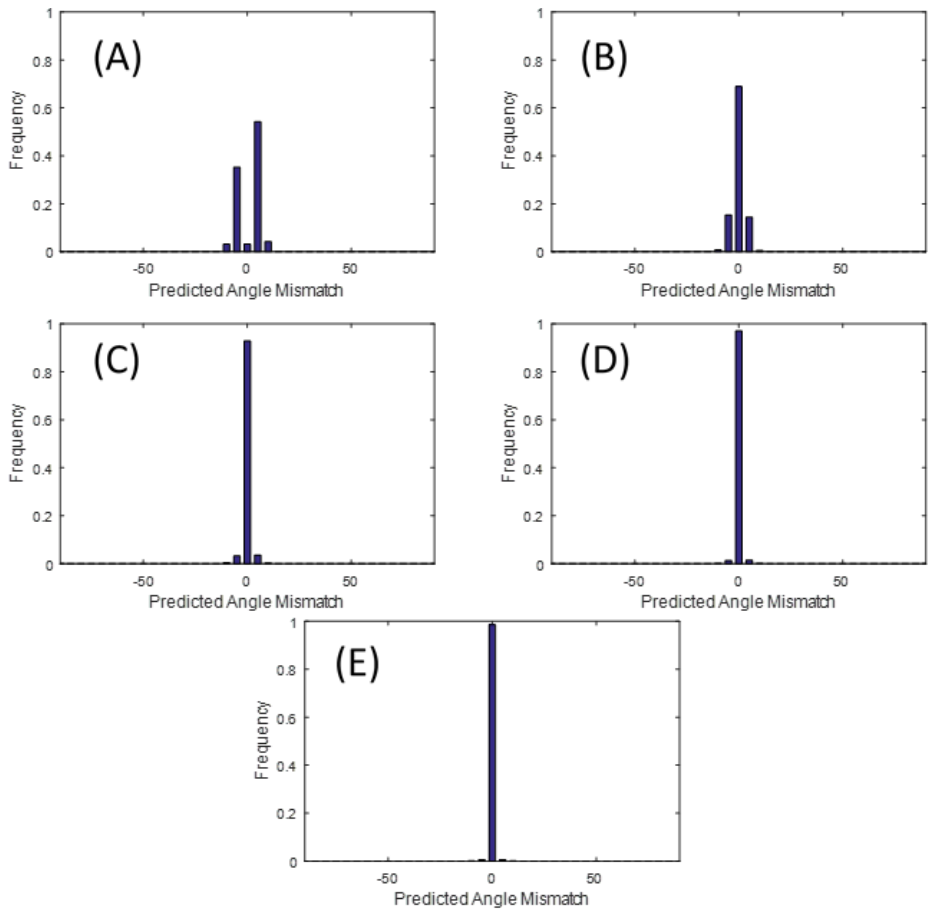

Figure 4: Distribution of the difference between the predicted initial grain boundary tilt angle and the actual angle calculated using the LDA. (A)-(E): Data base sizes of 10, 20, 30, 40 and 50 MD simulations per angle, respectively. introduced.[5-12] Recognizing this need, as well as a current trend in combining statistical and materials analysis,[33] we have explored the use of an image analysis and a statistical technique to compare plastic damage profiles among MD simulations. For this study, the damage was created by straining bi-crystals containing symmetric tilt grain boundaries with different initial tilt angles. The accuracy of the methods were evaluated by determining how well the final configuration from a simulation could be matched to an initial tilt angle by comparing the damage to a data base of straining simulations. Two potentials were used, one representing $\mathrm{Cu}$ and one

representing $\mathrm{Ag}$, and two coarse-grained descriptors for different types of crystal damage were used, CSP and HS. The image analysis method used the largest element of CC matrices created between all of the other MD simulations in the data base to connect damage to tilt angle. The LDA takes a DFFT of the rank-transformed coarse-grained matrices, and connects a simulation to the initial angle using an estimate of the mean and standard deviation for each combination of DFFT term, angle, and material type generated from the data base. In general the CSP is a more accurate descriptor than HS for both analysis methods, and for data base sizes of about 30 or more MD simulations per tilt angle, the LDA does considerably better in predicting angle and material than the $\mathrm{CC}$ method. For example, at the largest data base size of $50 \mathrm{MD}$ simulations per tilt angle and using the average CSP values, the LDA predicts the exact angle and material type for $92 \%$ of the simulations, while the CC approach drops to $58 \%$. If the average HS is used instead of the average CSP, the LDA and CC prediction drop to $63 \%$ and $32 \%$, respectively. Unlike the CC where a single descriptor is used (the largest CC coefficient), many parameters are estimated in the LDA (e.g. the mean and variance for each combination of material, angle, and DFFT term). These estimates can be imprecise when there are a small number of samples, which may account for the poor predictive capability of the LDA for relatively small sample sizes that improves and stabilizes for larger data sets of MD simulations. 
These results point to a number of possible applications of this method, for example in quantifying how the range of damage for a set of strained systems may depend on strain rate or temperature, or quantifying similarities between complex damage from processes such as indentation[13,34-37] and the interaction of energetic ions with solids[38-42] in terms of materials and damage conditions.

All of the molecular simulations and a majority of the analyses reported here were carried out by DL. BJR developed the LDA formalism and the initial code. DWB and BJR developed the initial concepts, and DWB wrote the majority of the manuscript in association with the other authors.

Graduate student and other financial support for this work was provided by the National Science Foundation through grant DMR1207145. A majority of the calculations were carried out on the NC State high-performance computing cluster, with support from the university.
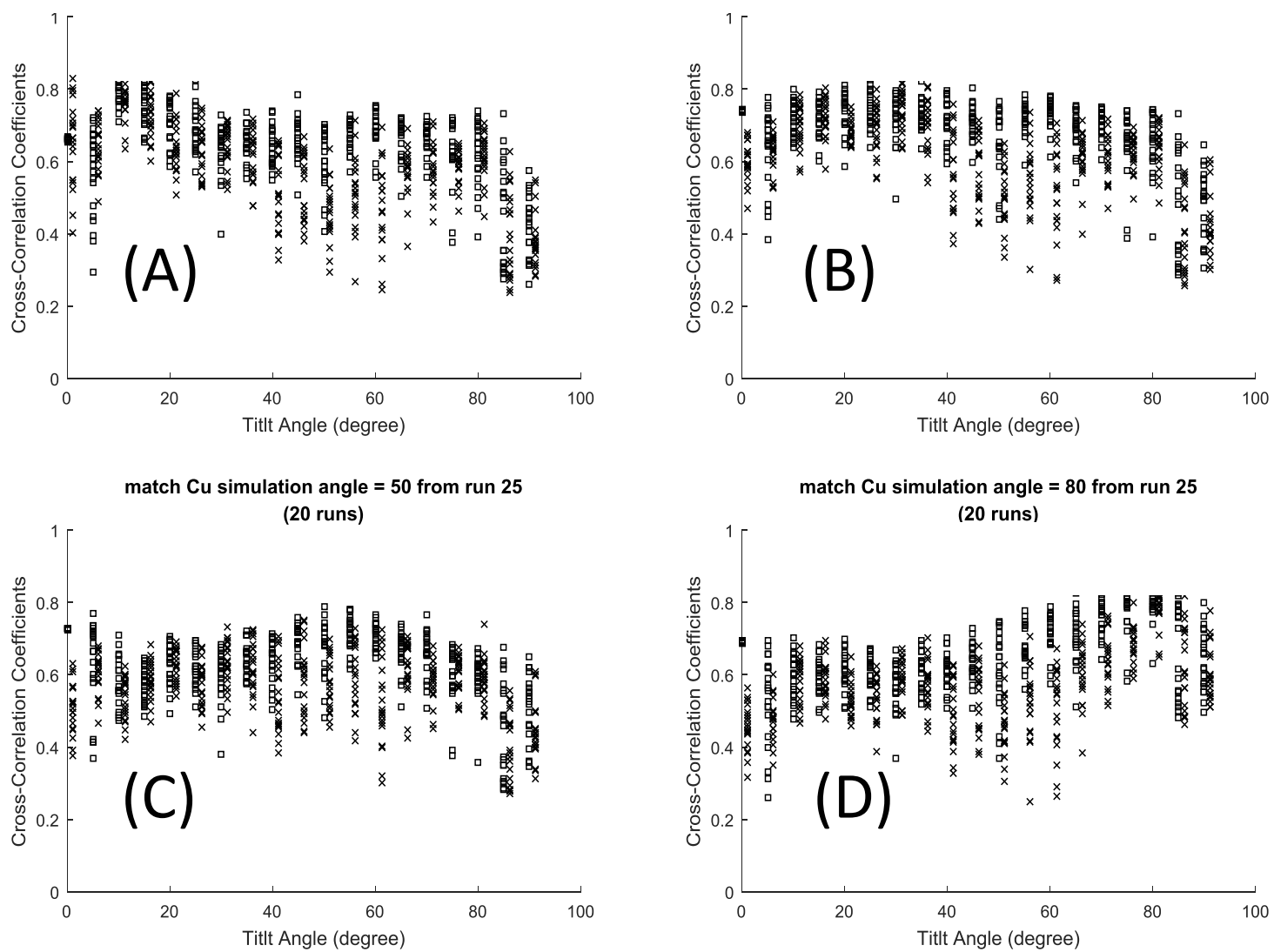

Figure 5: Largest element from each CC matrix generated using four example MD simulations with the Cu potential as a function of the tilt angle of the simulations to which each of the four simulations are correlated. These were generated using the data base of 20 runs per angle for each element. The squares correspond to a Cu run; the crosses correspond to a $\mathrm{Ag}$ run. (A)-(D): Example MD simulation with an initial tilt angle of $10^{\circ}, 30^{\circ}, 50^{\circ}$ and $80^{\circ}$, respectively. 


\section{References:}

[1] S. Lu, D. Li, D.W. Brenner, Molecular Dynamics Simulations of Plastic Damage in Metals, in: G.Z. Voyiadjis (Ed.), Handb. Damage Mech. Nano- to Macro- Scale Mater. Struct., Springer-Verlag, New York, 2015: pp. 453-486.

[2] A.M. Dongare, A.M. Rajendran, B. Lamattina, D.W. Brenner, M.A. Zikry, Atomic-scale study of plastic-yield criterion in nanocrystalline $\mathrm{Cu}$ at high strain rates, Metall. Mater. Trans. A Phys. Metall. Mater. Sci. 41 (2010) 523-531. doi:10.1007/s11661-009-0113-x.

[3] A.M. Dongare, A.M. Rajendran, B. Lamattina, M.A. Zikry, D.W. Brenner, Atomic scale simulations of ductile failure micromechanisms in nanocrystalline $\mathrm{Cu}$ at high strain rates, Phys. Rev. B - Condens. Matter Mater. Phys. 80 (2009) 1-11.

doi:10.1103/PhysRevB.80.104108.

[4] A.M. Dongare, B. LaMattina, A.M. Rajendran, Atomic scale studies of spall behavior in single crystal Cu, Procedia Eng. 10 (2011) 3636-3641. doi:10.1016/j.proeng.2011.04.598.

[5] K.G. Steenbergen, N. Gaston, Two worlds collide: image analysis methods for quantifying structural variation in cluster molecular dynamics., J. Chem. Phys. 140 (2014) 064102. doi:10.1063/1.4864753.

[6] M.T. Barakat, P.M. Dean, Molecular structure matching by simulated annealing. III. The incorporation of null correspondences into the matching problem, J. Comput. Aided. Mol. Des. 5 (1991) 107-117. doi:10.1007/BF00129750.

[7] J. Mestres, D.C. Rohrer, G.M. Maggiora, MIMIC: A Molecular-Field Matching Program. Exploiting Applicability of Molecular Similarity Approaches, J. Comput. Chem. 18 (1997) 934-954. doi:http://dx.doi.org/10.1002/(SICI)1096-987X(199705)18:7<934::AIDJCC6>3.0.CO;2-S.

[8] J.W.M. Nissink, M.L. Verdonk, J. Kroon, T. Mietzner, G. Klebe, Superposition of molecules: Electron density fitting by application of fourier transforms, J. Comput. Chem. 18 (1997) 638-645. doi:10.1002/(SICI)1096-987X(19970415)18:5<638::AIDJCC5>3.0.CO;2-O.

[9] X. Girones, D. Robert, R. Carbo-Dorca, TGSA: A molecular superposition program based on topo-geometrical considerations, J. Comput. Chem. 22 (2001) 255-263. doi:10.1002/1096-987x(20010130)22:2<255::aid-jcc12>3.3.co;2-u.

[10] B.L. Mooney, L.R. Corrales, A.E. Clark, MoleculaRnetworks: An integrated graph theoretic and data mining tool to explore solvent organization in molecular simulation, J. Comput. Chem. 33 (2012) 853-860. doi:10.1002/jcc.22917.

[11] B.L. Mooney, L.R. Corrales, A.E. Clark, Novel analysis of cation solvation using a graph theoretic approach, J. Phys. Chem. B. 116 (2012) 4263-4275. doi:10.1021/jp300193j.

[12] A. Ozkanlar, A.E. Clark, ChemNetworks: A complex network analysis tool for chemical systems, J. Comput. Chem. 35 (2014) 495-505. doi:10.1002/jcc.23506. 
[13] C.L. Kelchner, S.J. Plimpton, J.C. Hamilton, Dislocation nucleation and defect structure during surface indentation, Phys. Rev. B. 58 (1998) 11085-11088.

doi:10.1103/PhysRevB.58.11085.

[14] A. Stukowski, Structure identification methods for atomistic simulations of crystalline materials, Model. Simul. Mater. Sci. Eng. 20 (2012) 45021-15. doi:10.1088/09650393/20/4/045021.

[15] J.D. Honeycutt, H.C. Andersen, Molecular Dynamics Study of Melting and Freezing of Small Lennard- Jones Clusters, J. Phys. Chem. 91 (1987) 4950-4963. doi:10.1021/j100303a014.

[16] Y. Shi, M.L. Falk, Strain localization and percolation of stable structure in amorphous solids, Phys. Rev. Lett. 95 (2005) 1-4. doi:10.1103/PhysRevLett.95.095502.

[17] J.F. Panzarino, T.J. Rupert, Tracking microstructure of crystalline materials: A postprocessing algorithm for atomistic simulations, Jom. 66 (2014) 417-428.

doi:10.1007/s11837-013-0831-9.

[18] J.F. Panzarino, J.J. Ramos, T.J. Rupert, Quantitative tracking of grain structure evolution in a nanocrystalline metal during cyclic loading, Model. Simul. Mater. Sci. Eng. 23 (2015) 025005. doi:10.1088/0965-0393/23/2/025005.

[19] A. Stukowski, V. V Bulatov, A. Arsenlis, Automated identification and indexing of dislocations in crystal interfaces, Model. Simul. Mater. Sci. Eng. 20 (2012) 085007. doi:10.1088/0965-0393/20/8/085007.

[20] A. Stukowski, A. Arsenlis, On the elastic-plastic decomposition of crystal deformation at the atomic scale, Model. Simul. Mater. Sci. Eng. 20 (2012) 035012. doi:10.1088/09650393/20/3/035012.

[21] J. Hua, A. Hartmaier, Determining Burgers vectors and geometrically necessary dislocation densities from atomistic data, Model. Simul. Mater. Sci. Eng. 18 (2010) 045007. doi:10.1088/0965-0393/18/4/045007.

[22] G.C.L. Peterson, D. Li, J. Reich, D. Brenner, Spatial prediction of crystalline defects observed in molecular dynamic simulations of plastic damage, J. Appl. Stat. in press (2016).

[23] D. Li, E.W. Bucholz, G.C.L. Peterson, B.J. Reich, J.C. Russ, D.W. Brenner, How Predictable is Plastic Damage at the Atomic Scale?, Appl. Phys. Lett. in press. (2017).

[24] T. Hastie, R. Tibshirani, J. Friedman, The Elements of Statistical Learning: Prediction, Inference and Data Mining, Second Edi, Springer Verlag, 2009.

[25] S. Plimpton, Fast Parallel Algorithms for Short-Range Molecular Dynamics, J. Comput. Phys. 117 (1995) 1-19. doi:10.1006/jcph.1995.1039.

[26] Y. Mishin, M. Mehl, D. Papaconstantopoulos, A. Voter, J. Kress, Structural stability and lattice defects in copper: Ab initio, tight-binding, and embedded-atom calculations, Phys. 
Rev. B. 63 (2001) 1-16. doi:10.1103/PhysRevB.63.224106.

[27] P.L. Williams, Y. Mishin, J.C. Hamilton, An embedded-atom potential for the $\mathrm{Cu}-\mathrm{Ag}$ system, Model. Simul. Mater. Sci. Eng. 14 (2006) 817-833. doi:10.1088/09650393/14/5/002.

[28] S. Nosé, A unified formulation of the constant temperature molecular dynamics methods, J. Chem. Phys. 81 (1984) 511-519. doi:10.1063/1.447334.

[29] S. Nosé, A molecular dynamics method for simulations in the canonical ensemble, Mol. Phys. 52 (1984) 255-268. doi:10.1080/00268978400101201.

[30] S. Melchionna, G. Ciccotti, B. Lee Holian, Hoover NPT Dynamics for Systems Varying in Shape and Size, Mol. Phys. 78 (1993) 533-544. doi:10.1080/00268979300100371.

[31] J.C. Russ, F.B. Neal, The Image Processing Handbook, 7th Editio, CRC Press, Boca Raton, 2015.

[32] J.-P. Hansen, I.R. McDonald, Theory of Simple Liquids, 4th ed., Academic Press, Oxford, 2013.

[33] C.M. Fancher, Z. Han, I. Levin, K. Page, B.J. Reich, R.C. Smith, A.G. Wilson, J.L. Jones, Use of Bayesian Inference in Crystallographic Structure Refinement via Full Diffraction Profile Analysis, Sci. Rep. 6 (2016) 31625. doi:10.1038/srep31625.

[34] W. Yang, G. Ayoub, I. Salehinia, B. Mansoor, H. Zbib, Deformation mechanisms in Ti/TiN multilayer under compressive loading, Acta Mater. 122 (2017) 99-108.

doi:10.1016/j.actamat.2016.09.039.

[35] T. Fu, X. Peng, C. Wan, Z. Lin, X. Chen, N. Hu, Z. Wang, Molecular dynamics simulation of plasticity in VN(001) crystals under nanoindentation with a spherical indenter, Appl. Surf. Sci. 392 (2017) 942-949. doi:10.1016/j.apsusc.2016.09.130.

[36] C. Huang, X. Peng, T. Fu, Y. Zhao, C. Feng, Z. Lin, Q. Li, Nanoindentation of ultra-hard cBN films: A molecular dynamics study, Appl. Surf. Sci. 392 (2017) 215-224.

doi:10.1016/j.apsusc.2016.09.037.

[37] H. Dai, G. Chen, A molecular dynamics investigation into the mechanisms of subsurface damage and material removal of monocrystalline copper subjected to nanoscale high speed grinding, Appl. Surf. Sci. 303 (2014) 331-343. doi:10.1016/j.apsusc.2014.02.178.

[38] Y.N. Osetsky, A.F. Calder, R.E. Stoller, How do energetic ions damage metallic surfaces?, Curr. Opin. Solid State Mater. Sci. 19 (2015) 277-286. doi:10.1016/j.cossms.2014.12.001.

[39] M.J. Aliaga, R. Schäublin, J.F. Löffler, M.J. Caturla, Surface-induced vacancy loops and damage dispersion in irradiated Fe thin films, Acta Mater. 101 (2015) 22-30. doi:10.1016/j.actamat.2015.08.063.

[40] C. Björkas, K. Nordlund, Comparative study of cascade damage in Fe simulated with recent potentials, Nucl. Instruments Methods Phys. Res. Sect. B Beam Interact. with 
Mater. Atoms. 259 (2007) 853-860. doi:10.1016/j.nimb.2007.03.076.

[41] A.E. Sand, M.J. Aliaga, M.J. Caturla, K. Nordlund, Surface effects and statistical laws of defects in primary radiation damage: Tungsten vs. iron, EPL (Europhysics Lett. 115 (2016) 36001. doi:10.1209/0295-5075/115/36001.

[42] L.K. Beland, C. Lu, Y.N. Osetskiy, G.D. Samolyuk, A. Caro, L. Wang, R.E. Stoller, Features of primary damage by high energy displacement cascades in concentrated $\mathrm{Ni}$-based alloys, J. Appl. Phys. 119 (2016). doi:10.1063/1.4942533. 